\title{
PERSISTENT CURRENT IN A MESOSCOPIC RING WITH STRONGLY COUPLED POLARONS
}

\author{
M. Bayindir and I. O. Kulik \\ Department of Physics, Bilkent University \\ 06533 Bilkent, Ankara, Turkey
}

\begin{abstract}
We investigate influence of the electron-phonon interaction on persistent current in a mesoscopic ring threaded by a Aharonov-Bohm flux $\Phi$. We find that all thermodynamic quantities are still periodic in $\Phi$ with a period $\Phi_{0}=h c / e$. In both cases, weak- and strong-coupling, effective mass of the electrons is increased and amplitude of persistent current is suppressed. In the latter case the amplitude decreases exponentially as a function of the electron-phonon coupling constant.
\end{abstract}

\section{INTRODUCTION}

In classical electrodynamics, motion of a charged particle is completely determined by local electric field $\mathbf{E}$ and magnetic field $\mathbf{B}$ that act upon it. The scalar potential $\phi$ and vector potential $\mathbf{A}$ were first introduced as a mathematical tool for calculation concerning electromagnetic fields. However, in quantum theory these potentials appear in the Schrödinger equation explicitly and therefore they affect all physical quantities directly. Aharonov and Bohm [1] have shown that contrary to the conclusion of classical electrodynamics, there exist effects of the potentials on the charged particles, even in the region where all fields vanish (hence, there is no force acting on the particles). The experiment of Chambers [2] proved the existence of Aharonov-Bohm (AB) effect and stimulated experimental and theoretical studies in this field $[3,4,5]$. This effect has purely quantum mechanical origin because it comes from the interference phenomenon. The most well-known example of the $\mathrm{AB}$ effect is 
the persistent current in the normal-metal rings threaded by a magnetic flux $[6,7,8]$.

Sensitivity of the equilibrium properties, such as average energy or magnetization of a small free electron systems to a magnetic field was noticed rather early $[9,10,11]$. Existence of non-decaying (persistent) current in normal-metal rings (where mean free path $L_{\phi}$ exceeds the circumference of the ring $L=2 \pi R$ ) enclosing a magnetic flux was predicted by Kulik [6] in 1970. Later Büttiker, Imry, and Landauer [8] proposed the persistent current in the normal one-dimensional disordered ring. In 1990 's, experimental works $[12,13]$ confirmed the existence of persistent current in mesoscopic rings. This current arises due to the boundary conditions imposed on the wave function by the doubly connected nature of the loop. As a consequences of the boundary conditions, all physical properties of the ring are periodic in magnetic flux $\Phi$ with a period $\Phi_{0}=h c / e[14,15]$.

Scattering mechanisms result in decreasing, but not diminishing, of the persistent current. It is believed that inelastic, i.e. electron-phonon interaction, should be small to make the electronic states in the ring longlived (phase conserving). In the literature, the effects of the electronphonon interaction were studied in the mesoscopic $\mathrm{AB}$ rings in metallic $[16,17]$ and semiconducting cases [18].

In this paper we investigate the electron-phonon interaction within two limiting cases (weak- and strong-coupling) in a metallic $A B$ ring and show that the polaronic effect doesn't exclude the existence of flux quantization and persistent current in the ring.

\section{PERSISTENT CURRENTS IN MESOSCOPIC RINGS}

In this section we give a brief summary of the persistent current in mesoscopic $\mathrm{AB}$ rings in the absence of electron-phonon interaction (See Refs. $[19,20,21])$. Let us consider a one-dimensional ring with the circumference $L=N a$, where $N$ is the number of lattice sites and $a$ is the lattice constant ( $a=1$ is taken throughout this paper), threaded by a magnetic flux $\Phi$ as illustrated in Fig. 18.1.

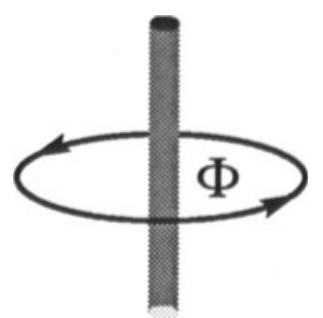

Figure 18.1 One-dimensional ring of circumference $L$ threaded by an AB flux $\Phi$. 
The existence of persistent current in the ring requires that there should not be strong localization, so that overlapping of the atomic orbitals is much important rather themselves. In the tight binding approximation, we can write the Hamiltonian as

$$
H_{e}=-t_{0} \sum_{n=1}^{N}\left(a_{n}^{+} a_{n+1} e^{i \alpha}+a_{n+1}^{+} a_{n} e^{-i \alpha}\right),
$$

where $t_{0}$ is hopping amplitude between the nearest-neighbors for an undistorted lattice, the operators $a_{n}\left(a_{n}^{+}\right)$annihilates (creates) an electron at site $n$ and $\alpha$ is the corresponding phase change which can be expressed in terms of $\mathrm{AB}$ flux $\Phi$

$$
\alpha=\frac{e}{\hbar c} \int_{n}^{n+1} \mathbf{A} \cdot d \mathbf{l}=2 \pi \frac{\Phi}{N \Phi_{0}},
$$

where $\Phi_{0}=h c / e \simeq 4.1 \times 10^{-7} \mathrm{G} \mathrm{cm}^{2}$ is the flux quantum. We can express the wave function in terms of the atomic orbitals:

$$
\Psi_{k}=\frac{1}{\sqrt{N}} \sum_{n=1}^{N} e^{i k n} a_{n}^{+}|0\rangle .
$$

where $|0\rangle$ is the vacuum state. From property of Fermi operators, $\left[a_{m}^{+}, a_{n}\right]_{+}=\delta_{m n}$, and the periodic boundary condition, the Schrödinger equation $H \Psi_{k}=\epsilon_{k} \Psi_{k}$ gives us

$$
\epsilon_{k}=-2 t_{0} \cos (k+\alpha), \quad k=\frac{2 \pi}{N} n, \quad n=0,1,2 \ldots N-1 .
$$

The corresponding eigenvalue spectrum and the persistent current are given by

$$
\begin{gathered}
\epsilon_{n}(\Phi)=-2 t_{0} \cos \left[\frac{2 \pi}{N}\left(n+\frac{\Phi}{\Phi_{0}}\right)\right], \\
I_{n}(\Phi)=-c \frac{d \epsilon_{n}}{d \Phi}=-I_{0} \sin \left[\frac{2 \pi}{N}\left(n+\frac{\Phi}{\Phi_{0}}\right)\right],
\end{gathered}
$$

where $I_{0}=4 c \pi t_{0} / N \Phi_{0}$ is the current amplitude. The energy is periodic in $\Phi$ with a period $\Phi_{0}$, so are all thermodynamic properties. The number of electrons, $\mathcal{N}$, is an obvious restriction for total energy which is the sum of all eigenvalues corresponding to an occupied state. Ground state energy (we ignore spin of the electron) is given by

$$
E(\Phi)=\sum_{n} \epsilon_{n}(\Phi)
$$




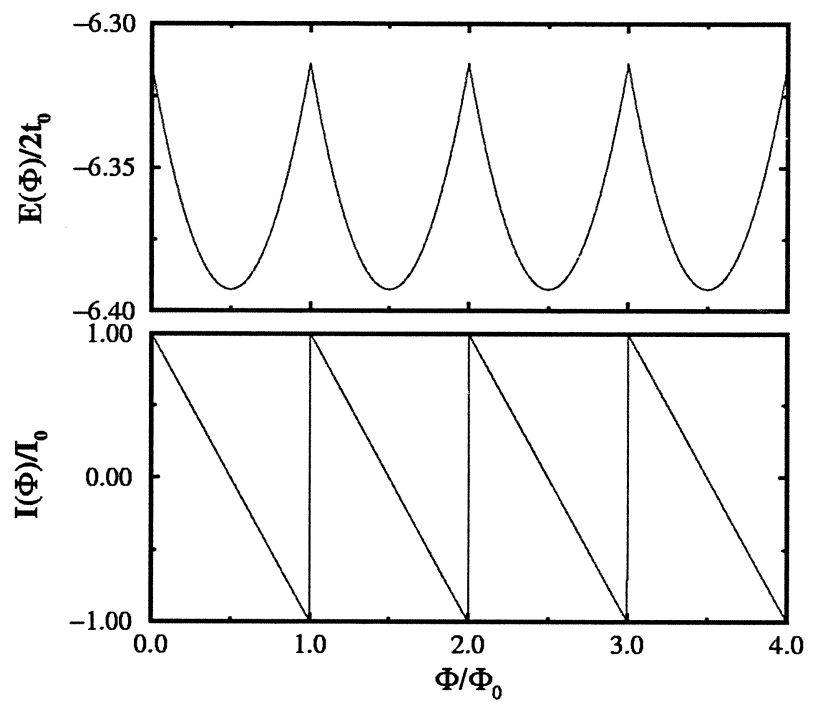

Figure 18.2 The normalized total energy $E(\Phi) / 2 t_{0}$ and the current $I(\Phi) / I_{0}$ as a function of flux $\Phi / \Phi_{0}$ for $N=20$ and $\mathcal{N}=10$ in a one-dimensional ring.

where the summation is carried out over $\mathcal{N}$ lowest energies for each value of flux $\Phi$. The total current flowing along the ring can be written as

$$
I(\Phi)=\sum_{n} I_{n}=-I_{0} \sum_{n} \sin \left[\frac{2 \pi}{N}\left(n+\frac{\Phi}{\Phi_{0}}\right)\right] .
$$

Figure 2 shows the periodic nature of the total energy and current as a function of flux $\Phi / \Phi_{0}$ for $N=20$ and $\mathcal{N}=10$ in the ring.

\section{ELECTRON-PHONON INTERACTION IN THE RING}

\subsection{Weak-Coupling Polarons}

The hopping amplitudes $t_{n, n+1}$ are changed when the atoms are displaced by $u_{n}$ from their positions in the undistorted lattice, and which can be expanded to the first order about the equilibrium position [22]:

$$
t_{n, n+1}=t_{0}+g\left(u_{n}-u_{n+1}\right),
$$

where $g$ is the electron-lattice displacement (electron-phonon) coupling constant. In present case, the Hamiltonian is modified as

$$
H=-\sum_{n=1}^{N} t_{n, n+1}\left(a_{n}^{+} a_{n+1} e^{i \alpha}+a_{n+1}^{+} a_{n} e^{-i \alpha}\right) \text {. }
$$


If we substitute the Eq. (18.9) into (18.10), the second term can be treated as the electron-phonon interaction Hamiltonian which is given by

$$
H_{e-p h}=g \sum_{n=1}^{N}\left(u_{n}-u_{n+1}\right)\left(a_{n}^{+} a_{n+1} e^{i \alpha}+a_{n+1}^{+} a_{n} e^{-i \alpha}\right),
$$

where $u_{n}$ can be written in terms of the phonon operators $b$ and $b^{\dagger}$

$$
u_{n}=\sum_{q} M_{q}\left(b_{q}+b_{-q}^{+}\right) e^{i q n}
$$

where $M_{q}=\left(\hbar / 2 M N w_{q}\right)^{1 / 2}, \omega_{q}=2 \omega_{0}|\sin (q / 2)|$ is the frequency of phonon with momentum $q$, and $b_{q}\left(b_{q}^{+}\right)$annihilates (creates) a phonon with momentum $q$. The operator $a_{n}$ can be written in the momentum representation as

$$
a_{n}=\frac{1}{\sqrt{N}} \sum_{k} a_{k} e^{i k n}
$$

By using Eqs. (18.12) and (18.13), $H_{e-p h}$ becomes

$$
\begin{aligned}
H_{e-p h}= & -g \sum_{k, q} M_{q}\left(1-e^{i q}\right)\left(b_{q}+b_{-q}^{+}\right) \\
& \times\left[e^{i(k+\alpha-q)} a_{k}^{+} a_{k-q}+e^{-i(k+\alpha+q)} a_{k+q}^{+} a_{k}\right] .
\end{aligned}
$$

For small values of coupling constant $g$, we can use the second order perturbation theory to determine contribution to the energy due to electronphonon interaction:

$$
\Delta E_{k}^{(2)}=\sum_{q} \frac{\left|\left\langle\phi_{0}\left|H_{e-p h}\right| \phi_{q}\right\rangle\right|^{2}}{E_{k}^{(0)}-E_{k+q}^{(0)}-\hbar \omega_{q}}
$$

where $\phi_{0}=a_{k}^{+}|0\rangle$ is the ground state and $\phi_{q}=a_{k+q}^{+} b_{-q}^{+}|0\rangle$ is the excited state, and $E_{k}^{(0)}=-2 t_{0} \cos (k+\alpha)$ are the energies of electron in the absence of electron-phonon interaction. Converting the summation in the Eq. (18.15) into integration, we get

$$
\Delta E_{k}^{(2)}=-\gamma \int_{-\pi / 2}^{\pi / 2} d q \frac{\cos ^{2}(p+q)}{\sin (p+q) \sin q /|\sin q|+\lambda}
$$

where $\gamma=\hbar g^{2} / \pi M t_{0} \omega_{0}$, and $\lambda=\hbar \omega_{0} / 2 t_{0}$ and $p=k+\alpha$. For small values of $p$, we can expand the integrand around $p=0$ by using the Taylor expansion formula

$$
\Delta E_{k}^{(2)}=-\gamma\left(J_{0}+J_{1} p+J_{2} p^{2} / 2+\cdots\right),
$$


where

$$
J_{0}=\int_{0}^{\pi / 2} d x \frac{2 \cos ^{2} x}{\sin x+\lambda}, \quad J_{1}=0, \quad J_{2}=\frac{1}{\lambda^{2}} .
$$

Hence, the second order correction to the energy due to electron-phonon coupling is given by

$$
\Delta E_{k}^{(2)}=-\gamma J_{0}-\frac{\gamma}{2 \lambda^{2}}(k+\alpha)^{2} .
$$

The total energy becomes

$$
\begin{aligned}
E_{k} & =E_{k}^{0}+\Delta E_{k}^{(2)} \\
& =E_{0}-2 t_{0} \cos (k+\alpha)-\frac{\gamma}{2 \lambda^{2}}(k+\alpha)^{2},
\end{aligned}
$$

where $E_{0}=-\gamma J_{0}$. For small values of $k+\alpha$, we can use the expansion $\cos (k+\alpha) \simeq 1-(k+\alpha)^{2} / 2$, then

$$
E_{k}=\tilde{E}_{0}+\frac{t^{\star}}{2}(k+\alpha)^{2},
$$

where $\tilde{E}_{0}=E_{0}-2 t_{0}$ and $t^{\star}=t_{0}\left(1-\gamma / 2 \lambda^{2} t_{0}\right)$. Since the effective mass of the electron is related to the hopping amplitude via $m^{\star} \propto 1 / t^{\star}$, we conclude that the effective mass of the electron is increased due to electron-phonon interaction:

$$
m^{\star}=m\left(1+\frac{\gamma}{2 \lambda^{2} t_{0}}\right) .
$$

In addition to the increasing of effective mass since $I_{0} \propto t^{\star}$, the amplitude of persistent current is suppressed due to electron-phonon coupling.

\subsection{Strong-Coupling Polarons}

We have studied the weak-coupling polarons in the previous section. In this section we will consider the strong-coupling case. The strong coupling polaron is the self-trapped state of the electron. If we denote by $l$ the characteristic size of the region which traps the electron (in reality, this region may move over the crystal as a whole), all of our calculations so far have related to case of large-radius polarons, $l \gg a$. We now consider small-radius polarons where $l \ll a$. The Hamiltonian of the system can be written as

$$
H=H_{e}+H_{p h}+H_{e-p h}
$$


where $H_{e}$ is the electronic part of the Hamiltonian which is given by the Eq. (18.1), $H_{p h}$ represents the phononic part which is given by

$$
H_{p h}=\sum_{q} \hbar \omega_{q} b_{q}^{+} b_{q}
$$

and $H_{e-p h}$ denotes the electron-phonon interaction which can be written as

$$
H_{e-p h}=\sum_{k, q} V_{q} a_{k+q}^{+} a_{k}\left(b_{q}+b_{-q}^{+}\right) .
$$

Using the Eq. (18.13), $H_{e-p h}$ can be written in the site representation as

$$
H_{e-p h}=\sum_{n, q} V_{q} a_{n}^{+} a_{n}\left(b_{q}+b_{-q}^{+}\right) e^{i q n} .
$$

where $V_{q}$ is the electron-phonon coupling constant which depends only on magnitude of $q$. We can take the wave function as

$$
\Psi=\sum_{m} C_{m} a_{m}^{+}|0\rangle
$$

where $C_{m}$ is a functions of the phonon operators. In the extreme strong-coupling limit one can neglect the hopping term $\left(t_{0}=0\right)$ in the Eq. (18.23). This approximation leads to a simplified equation

$$
\sum_{q}\left[\hbar \omega_{q} b_{q}^{+} b_{q}+V_{q}\left(b_{q}+b_{-q}^{+}\right) e^{i q m}\right] C_{m}=E C_{m}
$$

Introducing a transformation, $b_{q}=B_{q}+\Lambda_{q}$, in such a way that the electron-phonon interaction term in the Eq. (18.28) can be eliminated, yields

$$
\sum_{q}\left[\hbar \omega_{q} B_{q}^{+} B_{q}-\frac{V_{q}^{2}}{\hbar \omega_{q}}\right] C_{m}=E C_{m} .
$$

Notice that the operators $b_{q}$ and $B_{q}$ have the same commutation relations, $\left[b_{q}, b_{q^{\prime}}^{+}\right]=\delta_{q, q^{\prime}} \Longrightarrow\left[B_{q}, B_{q^{\prime}}^{+}\right]=\delta_{q, q^{\prime}}$, and $\Lambda_{q}=-\left(V_{q} / \omega_{q}\right) \exp (-i q m)$. The eigenvalues of the Eq. (18.29) are given by

$$
E^{(0)}=\sum_{q}\left(\hbar \omega_{q}-\frac{V_{q}^{2}}{\hbar \omega_{q}}\right) .
$$

Thus, the transformation leads to a decrease in phonon energy at each site by an amount $\Delta=V_{q}^{2} / \hbar \omega_{q}$, which is known as the polaron shift. For each values of $q$, the ground state wave function must satisfy the following relation:

$$
b_{q} \psi_{q}^{(0)}=\Lambda_{q} \psi_{q}^{(0)}
$$


The solution of the Eq. (18.31) is given by

$$
\psi_{q}^{(0)}=\exp \left(-\frac{1}{2}\left|\Lambda_{q}\right|^{2}\right) \sum_{n=0}^{\infty} \frac{\left(\Lambda_{q}\right)^{n}}{n !}\left(b_{q}^{+}\right)^{n}|0\rangle .
$$

The same result can be received with a canonical transformation of Lang and Firsov [23]. For all allowed values of $q$, the ground state wave function becomes

$$
\Psi_{m}^{(0)}=\prod_{q} \psi_{q}^{(0)}
$$

To understand the consequences of the strong electron-phonon coupling, we calculate matrix elements of $H_{e}$

$$
\left\langle\Psi_{n}^{(0)}\left|H_{e}\right| \Psi_{m}^{(0)}\right\rangle=-t^{\star} e^{i \alpha} \delta_{n, m+1}-t^{\star} e^{-i \alpha} \delta_{n, m-1} .
$$

Here, the hopping amplitude is modified as

$$
t^{\star}=t_{0} e^{-\Gamma}
$$

where

$$
\Gamma=2 \int_{0}^{\pi} \frac{d q}{2 \pi} \frac{V_{q}^{2}}{\hbar^{2} \omega_{q}^{2}}(1-\cos q) .
$$

Thus, in the strong-coupling case $(\Gamma \gg 1$ and $l \ll a)$, the hopping amplitude decreases exponentially as a function of electron-phonon coupling constant $V_{q}$. The effective mass increases exponentially because of $m^{\star} \propto 1 / t^{\star}$. More important result is that, in the present case, amplitude of the persistent current is suppressed exponentially since $I_{0} \propto t^{\star}=t_{0} e^{-\Gamma}$. Similar result was also obtained in Ref. [17] within a variational approach.

\section{CONCLUSIONS}

We investigated effects of electron-phonon interaction in the mesoscopic metal rings threaded by a magnetic flux. In both cases, weakor strong-coupling, in spite of the fact that magnitude of the persistent current is suppressed, the current is still periodic in flux $\Phi$ with a period $\Phi_{0}$. In the strong-coupling case, the magnitude is decreased exponentially as a function of the electron-phonon coupling strength. Finally, it is worth noting that the experiments were performed with a finite width rings [12]. Another effect of the electron-phonon interaction can be in the fluctuation pairing. This may increase $I_{0}$ since it was found quite early [24] that superconductivity restores due to Aharonov-Bohm effect in a small ring. The polaronic effect in coupled mesoscopic rings, as well as superconducting pairing and finite temperature effects will be subject of future investigation. 


\section{References}

[1] Y. Aharonov and D. Bohm, Phys. Rev. 115, 485 (1959).

[2] R. G. Chambers, Phys. Rev. Lett. 5, 3 (1960).

[3] A. Tonomura, Rev. Mod. Phys. 59, 639 (1987); Adv. Phys. 41, 59 (1992).

[4] M. Peshkin and A. Tonomura, The Aharonov-Bohm Effect (Springer, Berlin Heidelberg, 1989), and references therein.

[5] J. Hamilton, Aharonov-Bohm and Other Cyclic Phenomena (Springer, Berlin Heidelberg, 1997), and references therein.

[6] I. O. Kulik, Pis'ma Zh. Eksp. Teor. Fiz. 11, 407 (1970) [JETP Lett. 11, 275 (1970)].

[7] I. O. Kulik, in Quantum Optics and the Spectroscopy of Solids, edited by T. Hakioğlu and A. S. Shumovsky (Kluwer, Netherlands,1997), and references therein.

[8] M. Büttiker, Y. Imry, and R. Landauer, Phys. Lett. 96A, 365 (1983).

[9] L. Pauling, J. Chem. Phys. 4, 673 (1936).

[10] F. London, J. Phys. Rad. 8, 397 (1937).

[11] R. B. Dingle, Proc. Phys. Soc. A 212, 47 (1952).

[12] L. P. Lévy, G. Dolan, J. Dunsmuir, and H. Bouchiat, Phys. Rev. Lett. 64, 2074 (1990).

[13] V. Chandrasekhar, R. A. Webb, M. J. Brady, M. B. Ketchen, W. J. Gallagher, and A. Kleinsasser, Phys. Rev. Lett. 67, 3578 (1991).

[14] N. Byers and C. N. Yang, Phys. Rev. Lett. 7, 46 (1961).

[15] F. Bloch, Phys. Rev. B 2, 109 (1970).

[16] A. Ferretti, I. O. Kulik, and A. Lami, Phys. Rev. B 45, 5486 (1992).

[17] Yi-Chang Zhou, Xin-E Yang, and Huo-Zhong Li, Phys. Lett. 190A, 123 (1994).

[18] Tong-zhong Li, Ke-lin Wang, Gan Qin, and Jin-long Yang, J. Phys.: Condens. Matter 10, 319 (1998).

[19] S. Washburn and R. A. Webb, Adv. Phys. 35, 375 (1986).

[20] Y. Imry, Introduction to Mesoscopic Physics (Oxford University Press, New York-Oxford, 1997).

[21] Mesoscopic Physics and Electronics, edited by T. Ando et al. (Springer, Berlin Heidelberg, 1998).

[22] L. N. Bulaevskii, Ups. Fiz. Nauk. 115, 263 (1975) [Sov. Phys. Ups. 18, $131(1975)]$. 
[23] I. G. Lang and Yu. A. Firsov, Zh. Eksp. Teor. Fiz. 43, 1843 (1962) [Sov. Phys. JETP 16, 1301 (1963)].

[24] L. Gunther and Y. Imry, Solid State Commun. 7, 1391 (1969). 